\title{
28 Research Square \\ Cross Cultural Adaptation and Validation of a Spanish Version of VascuQol-6 Questionnaire.
}

\section{Barbara Soria-Juan}

Autonomous University of Madrid

\section{Lucía Llanos}

Jimenez Diaz Foundation University Hospital, FJD Health Research Institute, IIS-FJD UAM

Luis Riera Moral ( $\square$ luis.riera@salud.madrid.org )

Autonomous University of Madrid

\section{César Aparicio}

Autonomous University of Madrid

\section{Alejandro Gonzalez}

Jimenez Díaz Foundation University Hospital, FJD Health Research Institute, IIS-FJD UAM

\section{Oliver Shaw}

Jimenez Díaz Foundation University Hospital

Ignacio Mahillo Fernández

Jimenez Diaz Foundation University Hospital, FJD Health Research Institute, IIS-FJD-UAM

\section{Mariano Garcia-Arranz}

Jimenez Diaz Foundation University Hospital, FJD Health Research Institute, IIS-FJD UAM

\section{Research Article}

Keywords: Quality of Life, Health Related Quality of Life, VascuQol-6, Cross-cultural translation, Validation, Peripheral Arterial Disease.

Posted Date: December 6th, 2021

DOI: https://doi.org/10.21203/rs.3.rs-1135589/v1

License: (c) (1) This work is licensed under a Creative Commons Attribution 4.0 International License.

Read Full License 


\section{Abstract}

\section{Background:}

Outcomes in peripheral arterial disease (PAD) are usually clinical alone, ignoring patient's biopsychological sphere. VascuQol-6 is a specific questionnaire aimed to asses quality of life of patients suffering PAD. Our objective was to carry out a cross-cultural adaptation and validation of VQ-6 questionnaire from original English version into Spanish, in order to have a rapid, sensitive and easy-toapply clinical tool for evaluating HRQoL outcomes in PAD patients.

\section{Methods}

A prospective study with 100 outpatients with PAD patients was carried out in two university hospitals. Patients completed the Spanish translated VasquQol-6 (VQ-6) questionnaire and the SF-12 questionnaire. Validation of the VQ-6 Spanish Version was assessed through statistical measurement of reliability, reproducibility and validity of the questionnaire. Reproducibility of the Spanish VQ- 6 was assessed through Intraclass Correlation Coefficient, while Internal consistency was analyzed through Alpha Cronbach. Criterion Validity was assessed by comparing the SF-12 and with Pearson's Correlation Coefficient. Construct Validity was assessed by comparing the results of the questionnaire with the stage of the PAD based on Rutherford-Becker Category.

\section{Results}

On hundred patients were included in the study from July 2019 to January $2021.82 \%$ were male with a median age of 71.6 years. The reproducibility of the VQ-6 Spanish Version in each item was evaluated in a sample of 80 patients. Pain, Activity, Social and Emotional domains had ICC values ranging from 0.70 to 0.88 , indicating a good agreement. The value obtained for Cronbach's Alpha was 0.89 using all the questions of the questionnaire, indicating a good internal consistency. VQ- 6 and SF-12 showed positive correlations. The overall Pearson Correlation Coefficient was 0.77 for Physical SF-12, indicating an elevated Criterion Validity. Under's curve area was 0.8 , so discrimination capacity of VQ-6 Spanish Version to discriminate between IC and CLI was considered good.

\section{Conclusion}

VQ-6 Spanish Version is a reliable and valid instrument for evaluation of quality of life in patients suffering from PAD that can be used in clinical practice, adding a decision-base when evaluating different treatment options. This VQ-6 Spanish Version is intended to cover the spectrum of severity of PAD in Spanish-speaking patients.

Trial registration: ClinicalTrials.gov NCT04741321. Retrospectively registered on February 05,2021)

\section{Background}


Peripheral arterial disease (PAD) is the expression of atherosclerosis mainly in lower limbs that may prevent adequate arterial flow and lead to tissue ischemia. Clinical expression follows a chronological order, from an asymptomatic early stage to intermittent claudication (IC), pain at rest, and the presence of trophic wounds (1), resulting in critical limb ischemia (CLI). The therapeutic objectives pursued include healing of ischemic ulcers, relief of symptoms and improving quality of life of patients suffering this chronic and disabling condition.

HRQoL (Health-Related Quality of Life) questionnaires have been incorporated as a necessary measure tool in the evaluation of clinical outcomes, aimed to assess the impact of the disease and therapeutic options in patient's biopsychological sphere. Furthermore, they are of great relevance in the improvement of knowledge about different conditions $(2,3)$ and when analyzing the economic cost of an intervention. VascuQol is a specific questionnaire to assess the quality of life of patients suffering from PAD which is also used as an index in vascular registries (4). It was developed by Morgan et al. and published in 2001 (5). In the systematic review carried out by Poku et al. (6), in which different tools used for psychometric assessment were analyzed, VascuQol was recommended as the preferred questionnaire for measuring HRQoL outcomes in patients with PAD. The original version with 25 items (VascuQol-25) is divided into 5 subscales or domains including pain, symptoms, activity, social and emotional. The shortened version (VascuQol-6) was developed in order to facilitate the use of the tool (4), measuring the same domains. That questionnaire is made up of 6 questions with 4 possible answers, which score from 1 (worst quality of life perceived by the patient) to 4 (best quality of life perceived by the patient). The total score reaches from 6 to 24, and a higher value is related to a better health.

The validation process carried out by Larsen et al. (4) during the development of the VascuQol-6 questionnaire demonstrated greater precision and discrimination power to measure quality of life compared to the VascuQol-25 (VQ-25) questionnaire in a group of 21 patients with intermittent claudication. Nevertheless, while VQ-25 questionnaire has been translated and validated into 21 languages (7), the shortened version VascuQol-6 (VQ-6) is currently only available in a validated version in some languages $(4,8,9)$ not including Spanish. Hence, the main objective of this study was to carry out a cross-cultural adaptation and validation of VQ-6 questionnaire from original English version into Spanish, in order to have a rapid, sensitive and easy-to-apply clinical tool for evaluating HRQoL outcomes in patients with a diagnosis of PAD.

\section{Methods}

Translation, cross-cultural adaptation and validation of the questionnaire from original English version into Spanish was carried out using the methodology proposed by Ramada Rodilla et al (10).

\section{Participants}

A prospective study with 100 out patients (cohort $1, n=20$; cohort $2, n=80$ ) was carried out. Patients were recruited from the outpatient clinic of Angiology and Vascular Surgery Departments from two University Hospitals in Madrid, Spain (Jiménez Díaz Foundation University Hospital and La Paz University Hospital). 
Eligibility criteria included: 1) patients over 18 years of age, 2) from both genders, 3) with a diagnosis of PAD (intermittent claudication or critical limb ischemia), 4) whose first language was Spanish, 5) without cognitive impairment or other alteration that could difficult understanding of the questionnaire, and 6) who signed the Informed Consent Form. Patient relevant clinical information, including gender, age, employment status, PAD risk factors and stage were registered in a specific case report form in a pseudonymized fashion.

\section{Translation and Cross-cultural adaptation of VascuQol-6 questionnaire from English to Spanish}

Direct translation: Two bilingual translators (MGA, BSJ), whose native language was Spanish, independently carried out a conceptual translation into Spanish of the original English version. One of the translators knew the objectives and concepts considered in the questionnaire, and had previous experience in technical translation of texts, while the other translator had no prior knowledge of the questionnaire and was unaware of the objectives of the study.

Synthesis version: The two translators compared their individual translations and agreed on the final result. A process report was written together with the preliminary VQ-6 Spanish Version questionnaire (Version 1.0). That questionnaire was administered to four patients in order to have an approach of the understandability of the tool.

Back translation: Preliminary VQ-6 Spanish Version (Version 1.0) was back-translated into English by two native English language translators $(P F, O S)$ in order to warrant that the concepts of the original version had been preserved.

Consolidation by Committee of Experts: The four authors of the translation and back translation, together with an expert in methodology and a health professional (LLJ), confirmed the semantic and idiomatic equivalence of the questionnaire that had been back-translated into English with the Preliminary VQ-6 Spanish Version (Version 1.0). The final version was renamed as "VQ-6 Spanish Version" (Version 2.0).

Test / Applicability / Feasibility of VascuQol-6 Spanish Version: The scope of this step was to study whether the VQ-6 Spanish Version (Version 2.0) was understandable for a bigger sample of the population to whom the questionnaire is directed. Considering the type of evaluation and following the methodological recommendations (11), the sample size was established in 20 patients (cohort 1). Participants completed the VQ-6 Spanish Version and were asked the following questions "Are the questions easy to understand? Do you find them relevant for your condition? Do you have any suggestions?"

\section{Validation of the VascuQol-6 Spanish Version}

In order to assess the degree in which the VQ-6 Spanish Version was capable of measuring without bias (i.e. reliability of the instrument) (12), reproducibility and internal consistency of the questionnaire was statistically evaluated. The ability of the questionnaire to measure the construct for which it has been designed without bias $(19,23)$ was evaluated through the criterion validity and construct validity. 
A new sample of patients (cohort 2, $n=80$ ) was asked to fill out the VQ-6 Spanish Version (Version 2.0) at two different moments with a 3-4 week interval. This period of time was considered short enough to assure that clinical change had not occurred but long enough to prevent recall of what participants had answered when they filled the questionnaire for the first time. Reproducibility of VQ-6 Spanish Version was assessed through the calculation of the Intraclass Correlation Coefficient (ICC) together with its $95 \%$ confidence interval $(95 \% \mathrm{Cl})$. Coefficients $>0.7$ represented a good reproducibility $(13,14)$.

\section{Internal consistency}

Cronbach's alpha was used to evaluate the total score as well as each item of the questionnaire by eliminating each one of the questions in order to assess if the elimination of any of these questions affected the internal consistency. A reliable HRQoL measure should center between 0.7 and 0.9 for group comparisons $(15,16)$.

\section{Criterion validity}

Defined criterion validity establishes the validity of the questionnaire by comparing it with the gold standard test (12), which in this case was the SF-12 questionnaire (17). SF-12 is a reduced version of the SF-36 questionnaire, easy to apply to assess the functional capacity of people over 14 years of age. It includes eight dimensions: physical role, body pain, mental health, general health, vitality, social function and emotional role. We used version 2 of the tool, in which 50 (with an SD of 10) is taken as the mean of the general population. Values above 50 were interpreted as best, while values below 50 were interpreted as worse than the mean. For each of the items in each dimension, the score ranges from 0 (the worst health for that dimension) to 100 (the best health for that dimension). Patients from cohort 2 also completed the SF-12 questionnaire at baseline and after 3-4 weeks, and the results of both quality of life scales were compared through the Pearson's correlation coefficient, together with the linear regression line. Coefficients $>0.7$ were considered acceptable (13).

\section{Construct validity}

In this study, we evaluated whether the VQ-6 Spanish Version was capable of differentiate intermittent claudication from critical ischemia. Rutherford-Becker (RB) category is used in patients with PAD to classify chronic arterial disease into clinical categories (category 0 : asymptomatic; category 1 : mild claudication; category 2: moderate claudication; category 3 : severe claudication; category 4: pain at rest; category 5: minor tissue loss; category 6; ulcer o gangrene). The specific uniliteral hypothesis $(13,14,16)$ that we proposed was that patients with intermittent claudication (RB categories 1 , 2,3 ) would have higher scores in VQ-6 Spanish Version, while patients with critical ischemia (RB categories 4, 5 and 6 ) would have lower scores according to the published data of the original version of VQ-6 $(4,5,18)$. 


\section{Statistical analyses}

Quantitative variables were described with mean and standard deviation since there were no strong asymmetries in their distribution. Qualitative variables were described by absolute and relative frequencies. Internal consistency was measured by Cronbach's alpha. The coefficient was calculated for all the items and deleting each of them. To evaluate the intra-observer reliability, the intraclass correlation coefficient between test and retest measures was calculated. Also, a Bland-Altman plot was made to study the differences between test and retest measures. Criterion validity was evaluated by calculating the Pearson's correlation coefficient between the VQ-6 domains and the SF-12 physical and mental dimensions. Scatter plots were used to visualize the relation between VQ-6 domains and SF-12 physical and mental dimensions. To study the ability of VQ- 6 scores to discriminate between patients with claudication and patients with ischemia, a ROC curve was constructed, and its area was calculated. To construct the ROC curve the Leave-one-out cross validation method was used. All statistical analyses were performed using R version 4.0.5 (R: A language and environment for statistical computing. $R$ Foundation for Statistical Computing, Vienna, Austria).

\section{Results}

\section{Participants}

On hundred patients were included in the study from July 2019 to January $2021.82 \%$ were male with a median age of 71.6 years; $83 \%$ of the patients had intermittent claudication (RB categories 1, 2 and 3 ) and $17 \%$ suffered from critical limb ischemia (RB categories 4,5 and 6 ). $83 \%$ of patients were current or previous smokers, and $74 \%$ suffered from hypertension (Table 1 ).

\section{Cross-cultural adaptation of VascuQol-6 questionnaire from English into Spanish}

No major incongruences were found when translating and adapting the English questionnaire into Spanish. Preliminary VQ-6 Spanish Version (Version 1.0) was understandable for the four patients from the synthesis version step. Only one of the participants from cohort 1 (pre-test phase) referred difficulties with the understandability of one of the questions of VQ-6 Spanish Version (Version 2.0) when explicitly asked by the investigator. Minor modifications to the questionnaire were made, e.g., difference between "severely" (original) and "very" (translation), or changing "range of activity" from the original version by "activity" in the translated version. As less than $15 \%$ of the patients found difficulties in the Spanish translation, the VQ-6 Spanish Version was considered understandable for the population to whom it is directed (10).

\section{Validation of VascuQol-6 questionnaire Spanish Version}

\section{Test-ReTest / Reproducibility / Intra-observer reliability}

Reproducibility refers to the repeatability of the instrument when administered to the same population at two different times (12). The reproducibility of the VQ-6 Spanish Version in each item was evaluated in a 
sample of 80 patients. Pain, Activity, Social and Emotional domains had ICC values ranging from 0.70 to 0.88 , indicating a good agreement (Table 2). Symptoms domain had an ICC of 0.64 , indicating a moderate level of agreement. The ICC of the complete VQ-6 Spanish Version was 0.88 , indicating a very good degree of agreement (Figure 1).

\section{Internal consistency}

Internal consistency was measured to assess the degree of interrelation and coherence (homogeneity) between the different items from VQ-6 Spanish Version $(12,15,19)$. The internal consistency of VQ-6 Spanish Version in each subscale was calculated in a sample of 100 patients. The value obtained for Cronbach's Alpha was 0.89 using all the questions of the questionnaire, indicating a good internal consistency. Table 3 shows the values obtained by eliminating each of the questions. All the values were between 0.86 and 0.89 , showing that there is no question whose elimination affects the internal consistency of the questionnaire.

\section{Criterion validity}

Criterion validity of the VQ-6 Spanish Version was evaluated in a sample of 100 patients. To assess the correlation between the scores of VQ-6 Spanish Version and SF-12 questionnaires, a scatter plot was made, and Pearson's correlation coefficient was calculated, together with the linear regression line (Figure 2 and Table 4). In general terms, VQ-6 and SF-12 had positive correlations. The overall Pearson Correlation Coefficient was 0.77 for Physical SF-12, indicating an elevated Criterion Validity. We found better correlations between the VQ-6 questionnaire and SF-12 questionnaire regarding the Social domain with PhysicalSF-12. However, Pearson's correlation coefficient of VQ-6 Spanish Version with Mental SF-12 was 0.53 , indicating a moderate correlation.

\section{Construct Validity:}

Construct validity is intended to measure whether the VQ-6 Spanish Version is capable of distinguishing the different degrees of a certain variable we are comparing it with. Construct validity was assessed through the instrument's ability to discriminate between patients suffering from IC and CLI, using Receiver Operating characteristic (ROC) curve analysis with the RB category at baseline. The area under the curve was 0.8 , so discrimination capacity of VQ-6 Spanish Version to discriminate between IC and CLI was considered good.

\section{Discussion}

So far, VQ-6 has not been translated or adapted into Spanish. As it is a fast and easy-to-use tool in the consultation, that has demonstrated its statistical validation to measure PAD in other versions of the same questionnaire (other languages), the need to carry out, on the one hand, a translation and crosscultural adaptation of the questionnaire from English to Spanish, and on the other hand, to demonstrate the statistical validity of this new version, was considered justified. Simple translation of a questionnaire 
can lead to misinterpretations due to language and cultural differences. Therefore, cross-cultural adaptation followed a systematized process (10) that ensured that semantic and linguistic differences were corrected. In order to accept that the VQ-6 questionnaire translated into Spanish was valid for the entire PAD population, study population had to be the same as the one in which the original questionnaire was validated. The VQ- 6 was validated in patients with intermittent claudication and critical lower limb ischemia $(4,5)$. No bias selection was detected during recruitment of participants considering the stage of the PAD.

For the questionnaire to be used in clinical practice, it was necessary to demonstrate (i) its reliability or ability to measure without errors (15), (ii) its reproducibility when administered with the same method to the same population at two different times. (16), and (iii) its validity or ability to measure the construct for which it has been designed $(16,17)$. A very good reproducibility and internal consistency was demonstrated for the VQ-6 Spanish Questionnaire. Those data are in accordance with the validation carried out by Morgan et al. (5) during the development of the VQ- 6 questionnaire, with a positive test-re test reliability and evidence por internal consistency, and with subsequent validations of the VQ-6 questionnaire $(4,20)$. We also demonstrated a good correlation between the two QoL measurement tools (SF-12 and VQ-6 Spanish Version). Patients with CLI had a lower score in VQ-6 Spanish Version, while patients with IC had a higher one, indicating a good capacity of discerning between different stages of PAD based on quality of life. A good correlation with domains SF-12 score and excellent responsiveness to changes were demonstrated. This study maintains, with respect to the original version and with other validation carried out, the psychometric properties of the VascuQoL-6 health-related quality of life questionnaire $(4,6,9,18,20)$.

\section{Conclusions}

The Institute of Medicine has defined quality of care as the degree in which health services for individuals and populations increase the likelihood of desired health outcomes and are consistent with current professional knowledge (21). However, traditionally healthcare measured outcomes often fail to correspond with how a patient is actually feeling, since they don't take into account minimizing disability and improving quality of life. These new dimensions or attributes that condition the quality perceived by the patient are part of a what we call Medicine in Value, which consists on Integrating patient-reported outcome measures in daily vascular practice is gaining importance in clinical practice (24). HR-QoL questionnaires have been incorporated as a necessary tool in order to developpe new person-centered tools with the aim of enhancing quality of healthcare, and is considered essential for improving the quality and cost effectiveness of services and interventions (25).

For a questionnaire to be a feasible tool in daily clinical practice, it needs to be short, understandable and easy to complete. VQ-6 Spanish Version (Annex I) is a reliable and valid instrument for evaluation of QoL in patients suffering from PAD that can be used in clinical practice, adding a decision-base when 
evaluating different treatment options. This VQ-6 Spanish Version is intended to cover the spectrum of severity of PAD in Spanish-speaking patients.

\section{Abbreviations}

ABI: Ankle-Brachial Index

CLI: Critical Limb Ischemia

DM: Diabetes Mellitus

HRQoL: Health-related Quality of Life

IC: Intermittent Claudication

ICC: Intraclass Correlation Coefficient

PAD: peripheral arterial disease

Qol: Quality of life

RB category Rutherford-Becker category

SF-12: Short-Form 12

SF-36: Short-Form 36

VQ-6: Vascular quality of life questionnaire-6

VQ-25: Vascular quality of life questionnaire-25

\section{Declarations}

\section{Ethical considerations}

The study protocol was approved by FJD Ethics Committee and La Paz University Hospital Ethics Committee (reference number PIC114-19_FJD, July 2019 and PI-399, February 2020) and performed in accordance with the Declaration of Helsinki. Written Informed Consent to participate was obtained from all participants. All information was registered in a pseudonymized manner and was treated strictly confidential, in accordance with current regulations (Organic Law 3/2018 of December 5, Protection of Personal Data and Guarantee of Digital Rights, Regulation (EU) 2016/679 of the European Parliament and of the Council, of April 27, 2016 (GDPR), General Health Law 14/1986 and Law 14/2007 of Biomedical Research, Law 41/2002 of Patient Autonomy). 
Not applicable.

\section{Availability of data}

The anonymized data are available from the corresponding author if needed.

\section{Competing interests}

No potential conflict of interest is stated by the authors.

\section{Funding}

This study was carried out within the framework of the NOMA project funded by ISCIII (Carlos III Health Institute; file number PIC 18/00010).

\section{Author's contributions}

BS-J wrote the study protocol, obtained the EC approval, performed study associated procedures and wrote the first version of the manuscript. LL collaborated in the design and writing of the study protocol and supervised the development of the study. MGA conceived the study and supervised its development. LR, CA and AG recruited study participants. OS performed translations of the questionnaire from the Spanish Version into English and checked the last version of the Spanish VQ-6. IM participated in defining statistical considerations of the design and analyzed the results, providing figures and tables form the manuscript. All authors reviewed and approved the final version of the manuscript.

\section{Acknowledgments}

The authors acknowledge patients and their families for participate in the study.

\section{References}

1. Serrano Hernando FJ, Martín Conejero A. Enfermedad arterial periférica: aspectos fisiopatológicos, clínicos y terapéuticos. Rev Esp Cardiol. 1 de septiembre de 2007;60(9):969-82.

2. Measuring Health-Related Quality of Life | Annals of Internal Medicine [Internet]. [citado 14 de septiembre de 2021]. Disponible en: https://www.acpjournals.org/doi/10.7326/0003-4819-118-8199304150-00009?url_ver=Z39.88-2003\&rfr_id=ori:rid:crossref.org\&rfr_dat=cr_pub\%20\%200pubmed

3. Schwartzmann L. CALIDAD DE VIDA RELACIONADA CON LA SALUD: ASPECTOS CONCEPTUALES. Ciencia y enfermería. diciembre de 2003;9(2):09-21.

4. Larsen ASF, Reiersen AT, Jacobsen MB, Kløw N-E, Nordanstig J, Morgan M, et al. Validation of the Vascular quality of life questionnaire -6 for clinical use in patients with lower limb peripheral arterial disease. Health and Quality of Life Outcomes. 22 de septiembre de 2017;15(1):184.

5. Morgan MB, Crayford T, Murrin B, Fraser SC. Developing the Vascular Quality of Life Questionnaire: a new disease-specific quality of life measure for use in lower limb ischemia. J Vasc Surg. abril de 
2001;33(4):679-87.

6. Poku E, Duncan R, Keetharuth A, Essat M, Phillips P, Woods HB, et al. Patient-reported outcome measures in patients with peripheral arterial disease: a systematic review of psychometric properties. Health Qual Life Outcomes. 24 de noviembre de 2016;14:161.

7. Vascular Quality of Life Questionnaire-6 facilitates health-related quality of life assessment in peripheral arterial disease - ScienceDirect [Internet]. [citado 12 de septiembre de 2021]. Disponible en: https://www.sciencedirect.com/science/article/pii/S074152141301776X?via\%3Dihub

8. VASCUQOL - Vascular Quality of Life Questionnaire [Internet]. [citado 14 de septiembre de 2021]. Disponible en: https://eprovide.mapi-trust.org/instruments/vascular-quality-of-life-questionnaire

9. de Almeida Correia M, Andrade-Lima A, Mesquita de Oliveira PL, Domiciano RM, Ribeiro Domingues WJ, Wolosker N, et al. Translation and Validation of the Brazilian-Portuguese Short Version of Vascular Quality of Life Questionnaire in Peripheral Artery Disease Patients with Intermittent Claudication Symptoms. Annals of Vascular Surgery. 1 de agosto de 2018;51:48-54.e1.

10. Ramada-Rodilla JM, Serra-Pujadas C, Delclós-Clanchet GL. Adaptación cultural y validación de cuestionarios de salud: revisión y recomendaciones metodológicas. Salud Pública de México. febrero de 2013;55(1):57-66.

11. Perneger TV, Courvoisier DS, Hudelson PM, Gayet-Ageron A. Sample size for pre-tests of questionnaires. Qual Life Res. 1 de enero de 2015;24(1):147-51.

12. Beaton DE, Bombardier C, Guillemin F, Ferraz MB. Guidelines for the process of cross-cultural adaptation of self-report measures. Spine (Phila Pa 1976). 15 de diciembre de 2000;25(24):3186-91.

13. Terwee CB, Bot SDM, de Boer MR, van der Windt DAWM, Knol DL, Dekker J, et al. Quality criteria were proposed for measurement properties of health status questionnaires. $\mathrm{J}$ Clin Epidemiol. enero de 2007;60(1):34-42.

14. Fitzpatrick R, Davey C, Buxton MJ, Jones DR. Evaluating patient-based outcome measures for use in clinical trials. Health Technol Assess. 1998;2(14):i-iv, 1-74.

15. Cronbach LJ. Coefficient alpha and the internal structure of tests. Psychometrika. 1951;16:297-334.

16. Morris C, Janssens A, Allard A, Thompson Coon J, Shilling V, Tomlinson R, et al. Informing the NHS Outcomes Framework: evaluating meaningful health outcomes for children with neurodisability using multiple methods including systematic review, qualitative research, Delphi survey and consensus meeting [Internet]. Southampton (UK): NIHR Journals Library; 2014 [citado 17 de octubre de 2021]. (Health Services and Delivery Research). Disponible en: http://www.ncbi.nlm.nih.gov/books/NBK259778/

17. A 12-Item Short-Form Health Survey: Construction of Scales a...: Medical Care [Internet]. [citado 13 de septiembre de 2021]. Disponible en: https://journals.Iww.com/lwwmedicalcare/Abstract/1996/03000/A_12_Item_Short_Form_Health_Survey_Construction.3.aspx

18. Mehta T, Venkata Subramaniam A, Chetter I, McCollum P. Assessing the validity and responsiveness of disease-specific quality of life instruments in intermittent claudication. Eur J Vasc Endovasc Surg. enero de 2006;31(1):46-52. 
19. Piault E, Doshi S, Brandt BA, Angün Ç, Evans CJ, Bergqvist A, et al. Linguistic validation of translation of the self-assessment goal achievement (saga) questionnaire from English. Health Qual Life Outcomes. 23 de abril de 2012;10:40.

20. Kumlien C, Nordanstig J, Lundström M, Pettersson M. Validity and test retest reliability of the vascular quality of life Questionnaire-6: a short form of a disease-specific health-related quality of life instrument for patients with peripheral arterial disease. Health Qual Life Outcomes. 29 de septiembre de 2017;15:187.

21. Understanding Quality Measurement [Internet]. [citado 4 de noviembre de 2021]. Disponible en: http://www.ahrq.gov/patient-safety/quality-resources/tools/chtoolbx/understand/index.html

22. Black N. Patient reported outcome measures could help transform healthcare. BMJ (Clinical research ed). 28 de enero de 2013;346:f167.

23. Smith PC, Street AD. On the uses of routine patient-reported health outcome data. Health Econ. febrero de 2013;22(2):119-31.

24. Norman GR, Wyrwich KW, Patrick DL. The mathematical relationship among different forms of responsiveness coefficients. Qual Life Res. junio de 2007;16(5):815-22.

25. Neale J, Vitoratou S, Finch E, Lennon P, Mitcheson L, Panebianco D, et al. DEVELOPMENT AND VALIDATION OF 'SURE': A PATIENT REPORTED OUTCOME MEASURE (PROM) FOR RECOVERY FROM DRUG AND ALCOHOL DEPENDENCE. Drug and Alcohol Dependence. agosto de 2016;165:159-67.

\section{Tables}

Table 1. Demographics and risk factors in the patient population. DM: Diabetes Mellitus; BMI: Body Mass Index. *Defined as Glomerular Filtrate Rate $<60 \mathrm{mg} / \mathrm{dl}$ 
$\begin{array}{ll}\text { Variables } & \text { Mean } \pm \text { SD or } \\ & \text { No. }(\%)(n=100)\end{array}$

Demographics

\begin{tabular}{ll}
\hline Age, years & $71.6 \pm 11.2$ \\
\hline Sex & \\
\hline Female & $82(82 \%)$ \\
\hline Male & $18(18 \%)$ \\
\hline Employment status & $9(9 \%)$ \\
Employed & \\
\hline Absence due to incapacity & $5(5 \%)$ \\
\hline Unemployed & $4(4 \%)$ \\
\hline Retired & $82(82 \%)$ \\
\hline
\end{tabular}

Risk factors

\begin{tabular}{ll}
\hline Regular smoking & $32(32 \%)$ \\
\hline Ex-smokers & $51(51 \%)$ \\
\hline Hypertension & $74(74 \%)$ \\
\hline Previous cerebrovascular disease & $12(12 \%)$ \\
\hline Previous cardiovascular disease & $40(40 \%)$ \\
\hline Type 1 DM & $4(4 \%)$ \\
\hline Type 2 DM & $48(48 \%)$ \\
\hline Hyperlipidemia & $80(80 \%)$ \\
\hline Chronic pulmonary disease & $22(22 \%)$ \\
\hline Kidney disease* & $21(21 \%)$ \\
\hline Left Ankle-brachial index & $0.67 \pm 0.23$ \\
\hline Right Ankle-brachial index & $0.72 \pm 0.25$ \\
\hline BMI (mean \pm SD) & $27.4 \pm 5.6$ \\
\hline
\end{tabular}

Table 2. Test-retest or Reproducibility of items in the VQ-6 Spanish version questionnaire. ICC: Intraclass Correlation Coefficient. 


\begin{tabular}{|llll|}
\hline Item & Domain & ICC & $95 \% \mathrm{Cl}$ \\
\hline Question 1 & Social & 0.71 & $0.60-0.79$ \\
\hline Question 2 & Symptoms & 0.65 & $0.52-0.75$ \\
\hline Question 3 & Activity & 0.74 & $0.64-0.82$ \\
\hline Question 4 & Emotional & 0.77 & $0.68-0.84$ \\
\hline Question 5 & Social & 0.71 & $0.59-0.79$ \\
\hline Question 6 & Pain & 0.76 & $0.66-0.83$ \\
\hline Overall & & 0.88 & $0.83-0.92$ \\
\hline
\end{tabular}

Table 3. Cronbach's alpha coefficient for VascuQol-6 questionnaire in Cohort 1 and Cohort 2. Each subscale (Pain, Symptoms, Activity, Social and Emotional) was found to have an excellent reliability

\begin{tabular}{|lll|}
\hline Item & Domain & Cronbach's Alpha \\
\hline Question 1 & Social & 0.88 \\
\hline Question 2 & Symptoms & 0.87 \\
\hline Question 3 & Activity & 0.86 \\
\hline Question 4 & Emotional & 0.87 \\
\hline Question 5 & Social & 0.87 \\
Question 6 & Pain & 0.87 \\
\hline Overall & & 0.89 \\
\hline
\end{tabular}

Table 4. Criterion validity. Pearson Correlation coefficient. 


\begin{tabular}{|lll|}
\hline VQ-6 Domain & SF-12 Physical & SF-12 Mental \\
\hline Social & 0.75 & 0.41 \\
\hline Symptoms & 0.56 & 0.47 \\
\hline Activity & 0.67 & 0.44 \\
\hline Emotional & 0.59 & 0.48 \\
\hline Pain & 0.60 & 0.49 \\
\hline Overall & 0.77 & 0.53 \\
\hline
\end{tabular}

Table 5. RB category.

\begin{tabular}{|lll|}
\hline $\begin{array}{l}\text { Rutherford-Becker } \\
\text { Category }\end{array}$ & $\mathrm{N}(\%)$ \\
\hline 1 & Mild claudication & $9(9 \%)$ \\
\hline 2 & Moderate claudication & $55(55 \%)$ \\
\hline 3 & Severe claudication & $19(19 \%)$ \\
\hline 4 & Rest pain & $2(2 \%)$ \\
\hline 5 & Ischemic ulcers (minor tissue loss) & $10(10 \%)$ \\
\hline
\end{tabular}

Figures 


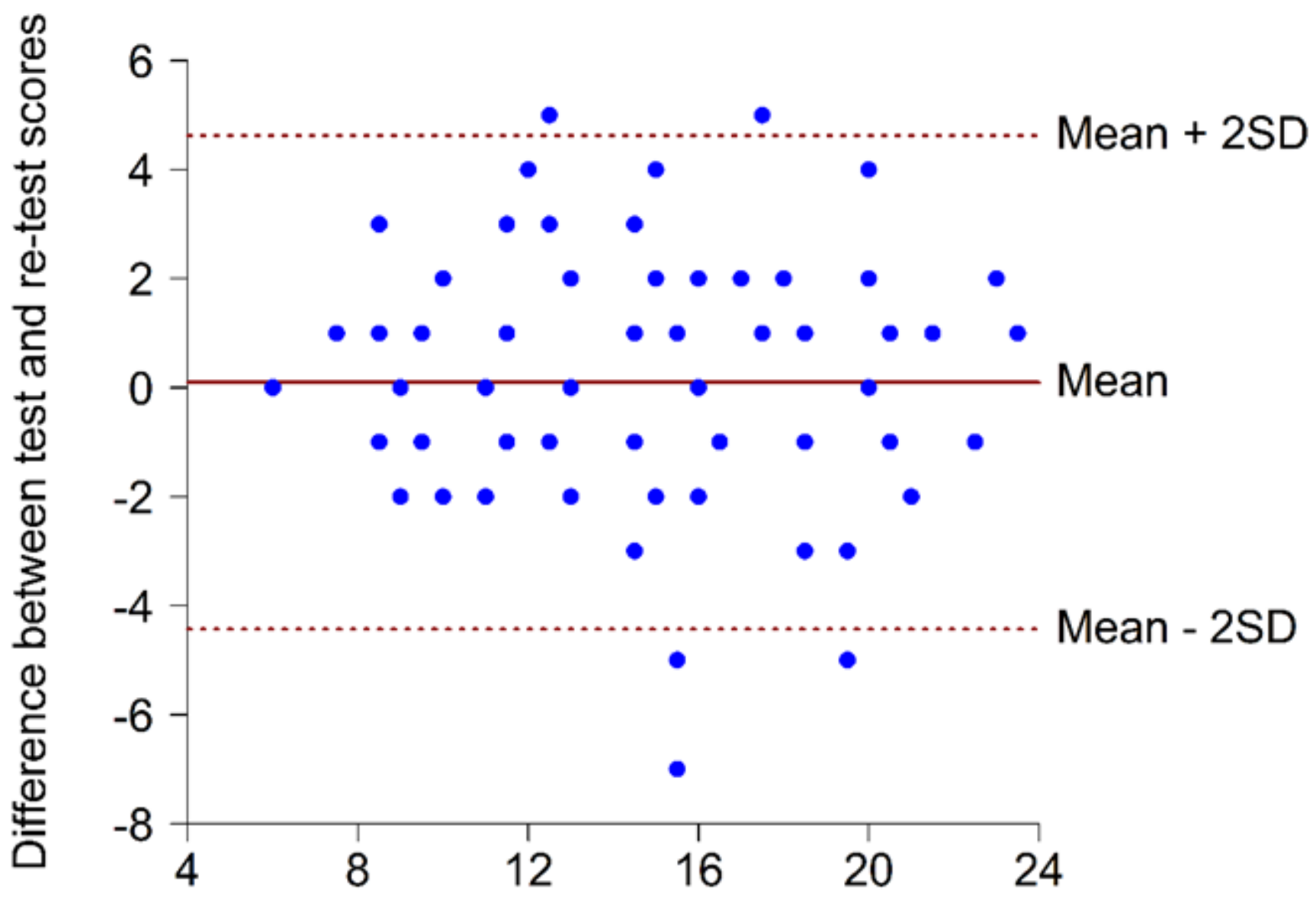

Average of test and re-test scores

Figure 1

Bland-Altman graphic. The differences between the test and retest scores are constant throughout the range of values of the VQ- 6 Spanish Version. The mean of the differences is practically zero, showing a great similarity between test and re-test scores. 
A
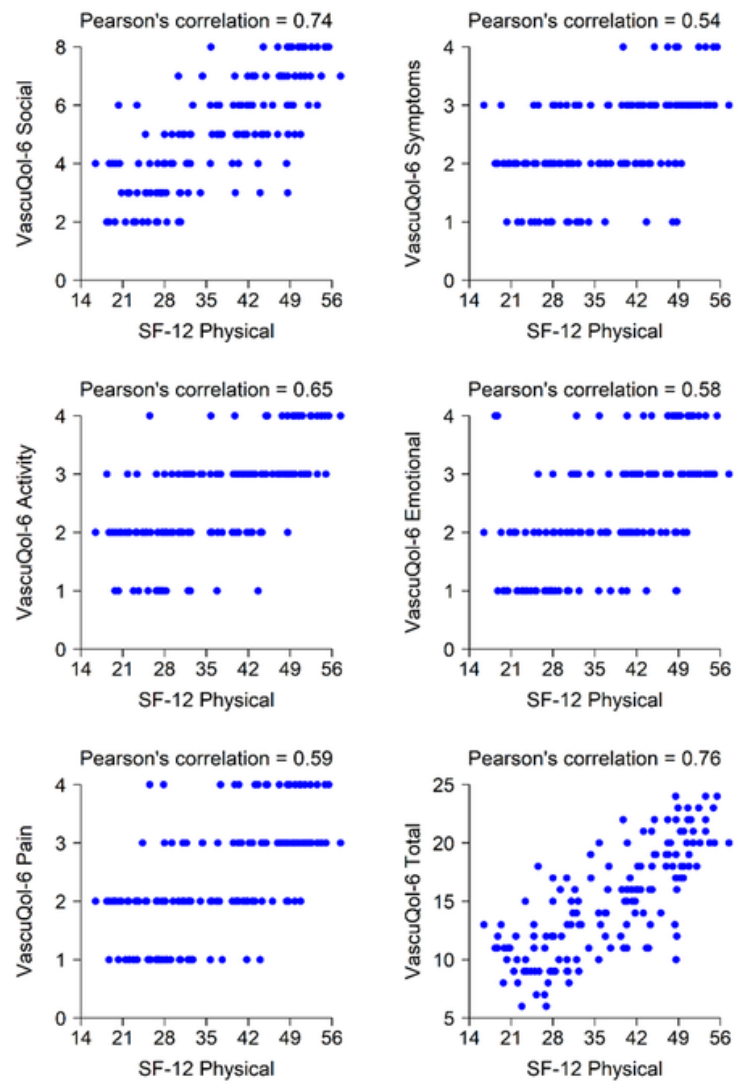

B
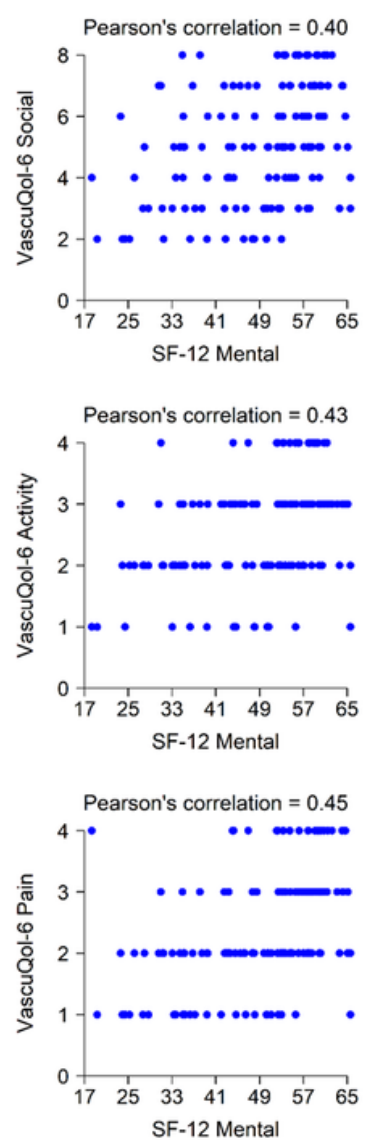
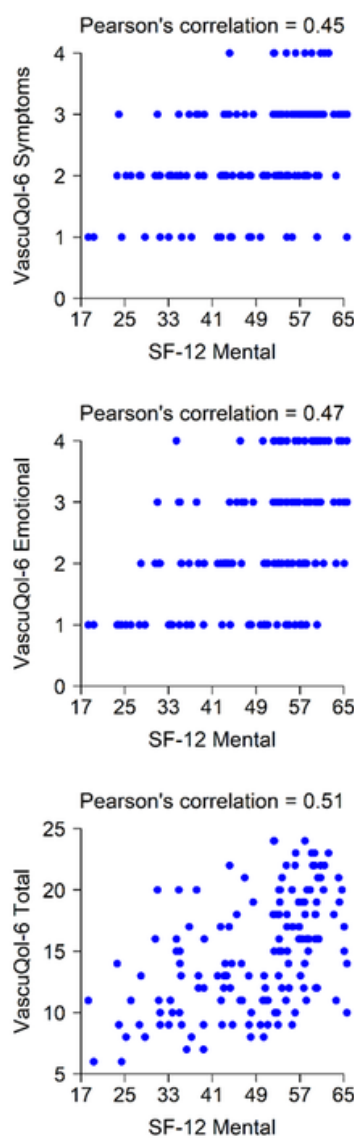

Figure 2

A. Criterion validity of VQ-6 with Physical SF-12. Scaller spot. VQ-6 and SF-12 had positive correlations. Higher values of SF-12 correspond to higher values of VQ-6. If the correlation coefficient is equal to or greater than 0.7 , we speak of a high correlation. If the coefficient is between 0.5 and 0.7 we find a moderate correlation. Below 0.5 we would speak of a low correlation. B. Criterion validity of VQ- 6 with Mental SF-12. Scaller spot. En cuanto a la curva ROC la he hecho juntando test y retest 


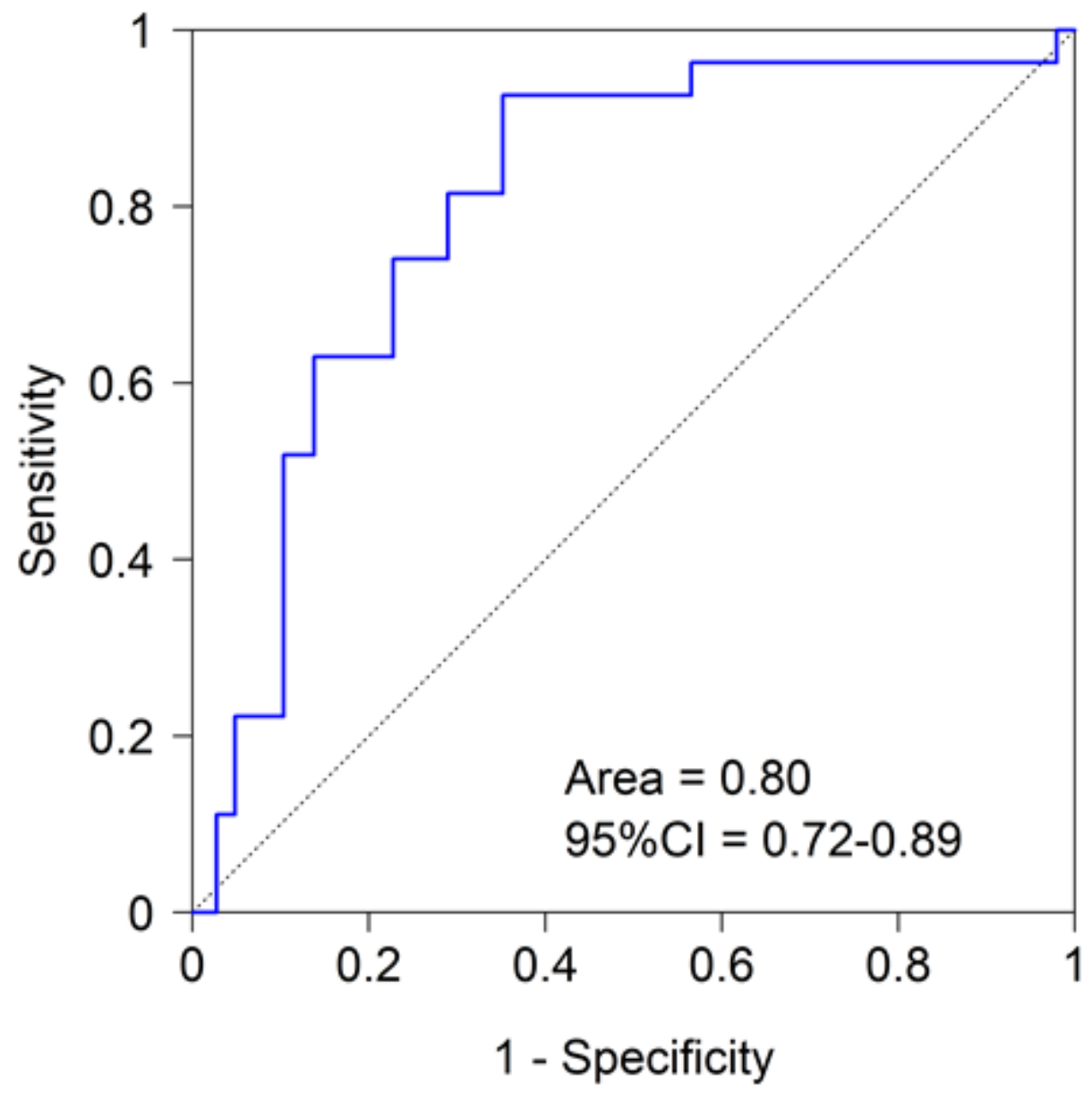

Figure 3

Construct validity of VQ-6 Spanish Version The area under the curve is 0.8 , so discrimination capacity of VQ-6 Spanish Version to discriminate between IC and CLI was considered good. 